\title{
The effects of fiber's surface roughness on the mechanical properties of fiber-reinforced polymer composites
}

Journal of Composite Materials 47(23) 2909-2923

(C) The Author(s) 2012

Reprints and permissions: sagepub.co.uk/journalsPermissions.nav DOI: | $0 . \mid$ | 177/002 | 9983 |245987| jcm.sagepub.com

@SAGE

\author{
Yin Yao and Shaohua Chen
}

\begin{abstract}
An improved shear-lag model is developed in this paper to study the effects of interface roughness on the mechanical properties of unidirectional fiber-reinforced polymer composites with a staggered structure, in which the roughness is incorporated by establishing equilibrium equations for the fiber platelets with varying thickness along its axial direction. The stress transfer and effective Young's modulus of composites are mainly investigated due to the influence of fiber's surface roughness. Since the polymer matrix can be chosen as thermoplastic or thermosetting materials, a uniformly interfacial shear stress distribution due to the frictional transfer along fiber/matrix interfaces and a non-uniformly one due to the elastic transfer are analyzed, respectively. It is found that when the surface roughness becomes larger, fibers in the former will carry more tensile loads, while the tensile loads keep almost invariant in fibers and the shear stress reduces in matrix in the latter. Moreover, the effective Young's modulus of composites will be enhanced due to increasing fiber's surface roughness. However, the enhancing effect will gradually reduce with an increasing aspect ratio of fibers. The results should be very useful for the design of novel fiber-reinforced polymer composites, especially for those that needed interfacial modifications in order to improve the interfacial adhesion, for example, carbon-fiber reinforced polymer composites.
\end{abstract}

\section{Keywords}

Fiber-reinforced polymer composites, interface roughness, shear-lag model, stress transfer, effective Young's modulus

\section{Introduction}

The performance of fiber-reinforced polymer composites is largely dependent on the properties of fiber/ matrix interface. Strong interfacial adhesion guarantees efficient load transfer from the soft organic matrix to stiff fibers with high strength and modulus, which helps improving the overall load bearing capacity of composite structures. However, fibers with inert surfaces (e.g. carbon, aramid and polyethylene) always achieve weak interfacial adhesion to the polymer matrix, ${ }^{1-4}$ leading to the failure of composites relevant to interface debonding. Hence, lots of surface modification techniques are developed in order to strengthen the fiber/ matrix interface, which is of great significance to the optimal design of high-quality composites. ${ }^{1-6}$

One of the surface modification techniques is to increase fiber's surface roughness with large number of micro-grooves and ridges produced by etching physically or chemically. ${ }^{5,7}$ As a result, the interfacial contact area gets enlarged and the mechanical interlocking between fiber and polymer matrix is enhanced to provide high bond strength. Therefore, interface roughness plays an important role in the strengthening of interfacial adhesions. Many experimental results have proved this aspect that the interfacial shear strength apparently increases with the augmentation of interface roughness. ${ }^{8-11}$ Additionally, some experimental researches show that the effective Young's modulus and strength of composites with reinforcements treated by surface etching are raised as compared to the untreated ones, ${ }^{9,12-14}$ which suggest that interface roughness also contributes to the enhancement of

Institute of Mechanics, Chinese Academy of Sciences, Beijing, China

\section{Corresponding author:}

Shaohua Chen, LNM, Institute of Mechanics, Chinese Academy of Sciences, Beijing, 100190, China.

Email: chenshaohua72@hotmail.com 

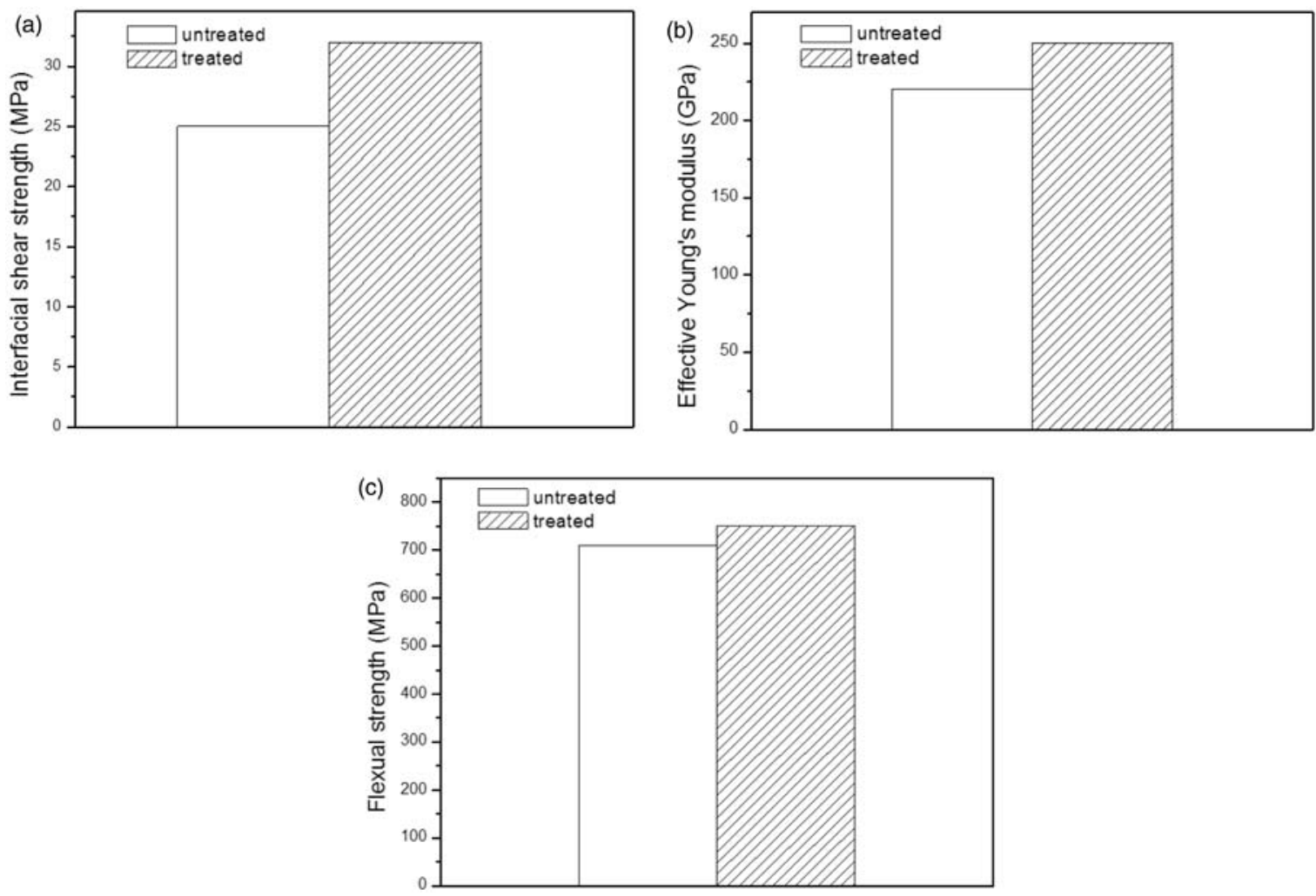

Figure I. Improved mechanical properties of carbon/polymer composites with fibers treated by surface etching ${ }^{11,12}$ : (a) interfacial shear strength (IFSS), (b) effective Young's modulus and (c) flexural strength.

overall mechanical properties. The improvements of these mechanical properties are schematically depicted in Figure 1. However, if the surface treatment lasts too long, the strength of composites will gradually decrease because the micro-pitting on the fiber surface will accumulate and grow into small defect, which will reduce the strength of fibers. ${ }^{10,11}$

Many attentions have been paid on fiber's surface roughness in fiber-reinforced polymer composites, but most of them are confined to experimental studies, as mentioned above. The existing theoretical researches mainly focus on the influence of interface roughness on the frictional sliding of fibers in pull-out and pushout processes. ${ }^{15-18}$ These works provided some basic approaches for describing the rough surface topography (e.g. Fourier series and trigonometric functions), based on which the interface roughness can be incorporated into theoretical models. However, the effects of interface roughness on the mechanical properties of composites during surface modification, such as the improved stress transfer at perfect interface and the enhanced overall stiffness, ${ }^{9,12-14}$ were seldom touched upon in the former theoretical works. Further researches on this issue are still necessary.

In the present paper, an improved shear-lag theoretical model is established to explore the influence mechanisms of interface roughness on stress transfer and effective Young's modulus in fiber-reinforced polymer composites with a staggered structure. Not only a frictional interface but also an elastic deformation interface between fibers and matrix will be considered, corresponding to the utilizations of thermoplastic and thermosetting polymer matrices in composites, respectively. ${ }^{19,20}$ Fiber's roughness is evaluated by the ratio of the micro-scale amplitude and wavelength of the rough surface. Analytical solutions considering the effect of interfacial roughness will be compared to those obtained for cases with a smooth interface. All the results should be helpful for the design of novel composites, especially, for those that needed interfacial modifications in order to improve the interfacial adhesion, for example, carbon-fiber reinforced polymer composites.

\section{Theoretical model and analysis}

\section{An improved shear-lag model}

Figure 2 plots a two dimensional (2-D) plane strain configuration of staggered fiber-reinforced polymer composites. Although a 3-D circular cylinder is a 


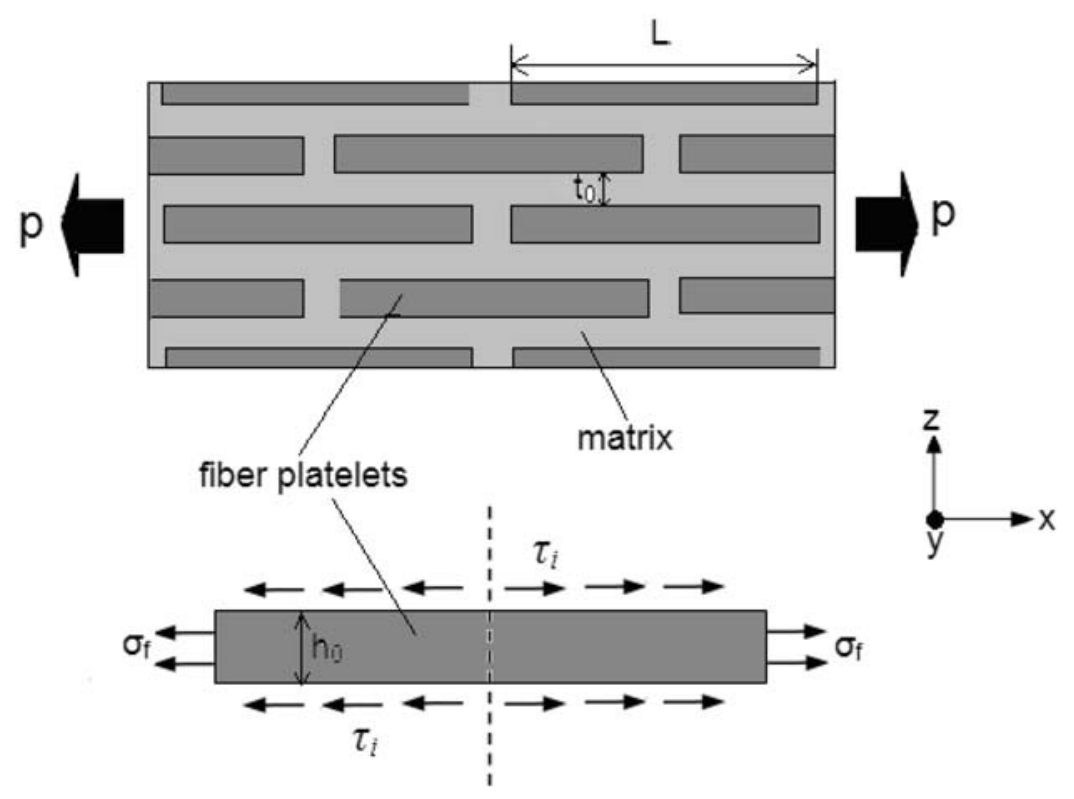

Figure 2. Two-dimensional pattern of staggered composites and an individual fiber platelet subjected to tensile stress and interfacial shear stress.

more preferred fiber configuration, ${ }^{21}$ the current model with 2-D fiber platelets is also effective and accurate for qualitative investigations of micromechanical properties of composites. ${ }^{22}$ In addition, the staggered structure is always adopted to characterize a novel bio-composite system (nacre, human bone, etc.), which consist of mineral platelets and protein polymer matrix. $^{23} \mathrm{We}$ assume that the distribution of fiber repeats itself after two fiber platelets, and the neighboring platelets overlap half of their length along longitudinal direction (regular staggering). ${ }^{24} h_{0}$ and $L$ are the thickness and length of each fiber platelet, respectively, and $t_{0}$ is the thickness of the polymer matrix in between the parallel fibers.

When a tension load $p$ is applied to the staggered composites, it is often considered that the stiff fiber platelets mainly carry the tensile stress and the soft polymer matrix carries shear stress. The tensile load is transferred from matrix to fibers via the interfacial shear stress $\tau_{i}$ distributed along the fiber/matrix interface. Since the thicknesses of fiber and matrix are much smaller than their lengths, the axial tensile strain and stress in fiber platelets are assumed to be dependent only on $x$ axis. Therefore, with the help of an infinitesimal fiber element with length $d x$ as shown in Figure 3, the equilibrium relation between the interfacial shear stress $\tau_{i}$ and axial stress $\sigma_{f}$ in fibers can be written as

$$
\left(\sigma_{f}+d \sigma_{f}\right) h_{0}+2 \tau_{i} \cdot d x=\sigma_{f} h_{0}
$$

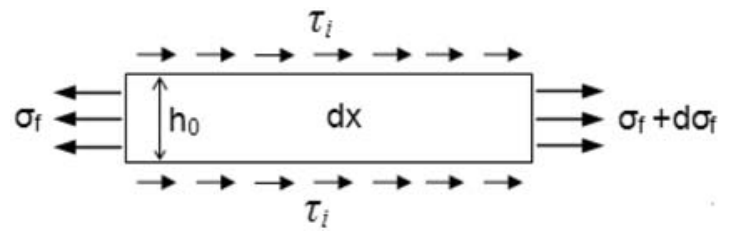

Figure 3. An infinitesimal fiber element.

Then, we have

$$
\frac{d \sigma_{f}(x)}{d x}=-\frac{2}{h_{0}} \tau_{i}(x)
$$

Equation (2) is the classical form of shear-lag equation (here the width in $y$ direction is assumed to be unity), ${ }^{25}$ which was used in the previous theoretical researches in order to analyze the effects of interface roughness. ${ }^{15,16}$ However, equation (2) is derived only for the case with a smooth interface. For the case with a rough interface, the wavy surface of fiber leads to a more complex boundary condition, which is analyzed as follows.

As shown in Figure 4(a), an infinitesimal fiber element is taken from the staggered composite structure with a rough fiber/matrix interface, whose contour line is illustrated in Figure 4(b). Similar to References $[18,26]$, the rough interface is set to be periodically wavy in $x$ direction, having a micro-scale amplitude $\Delta$ and a wavelength $\lambda \cdot{ }^{10,16}$ Consequently, the thickness 


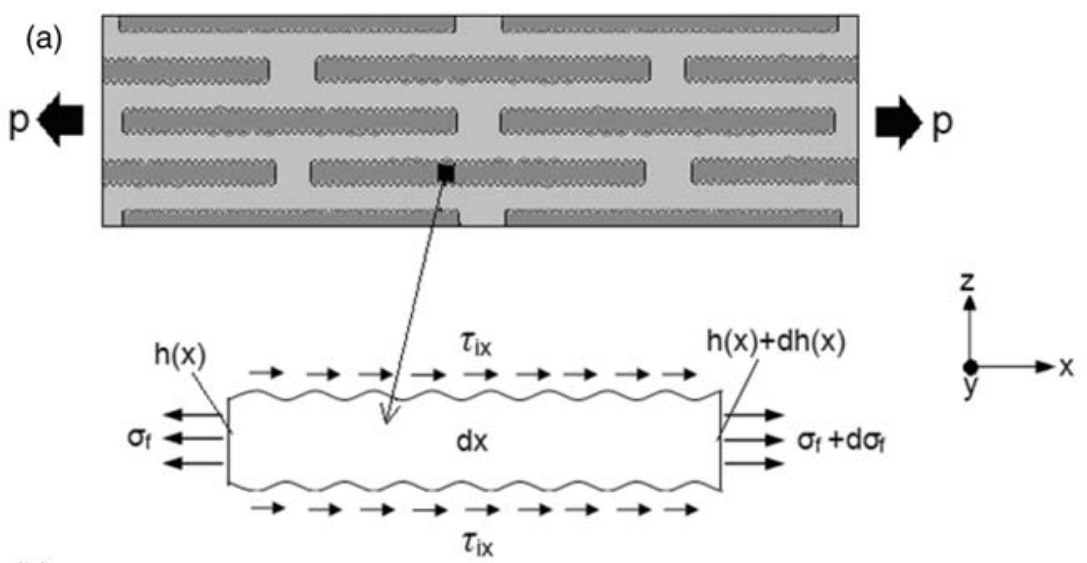

(b)

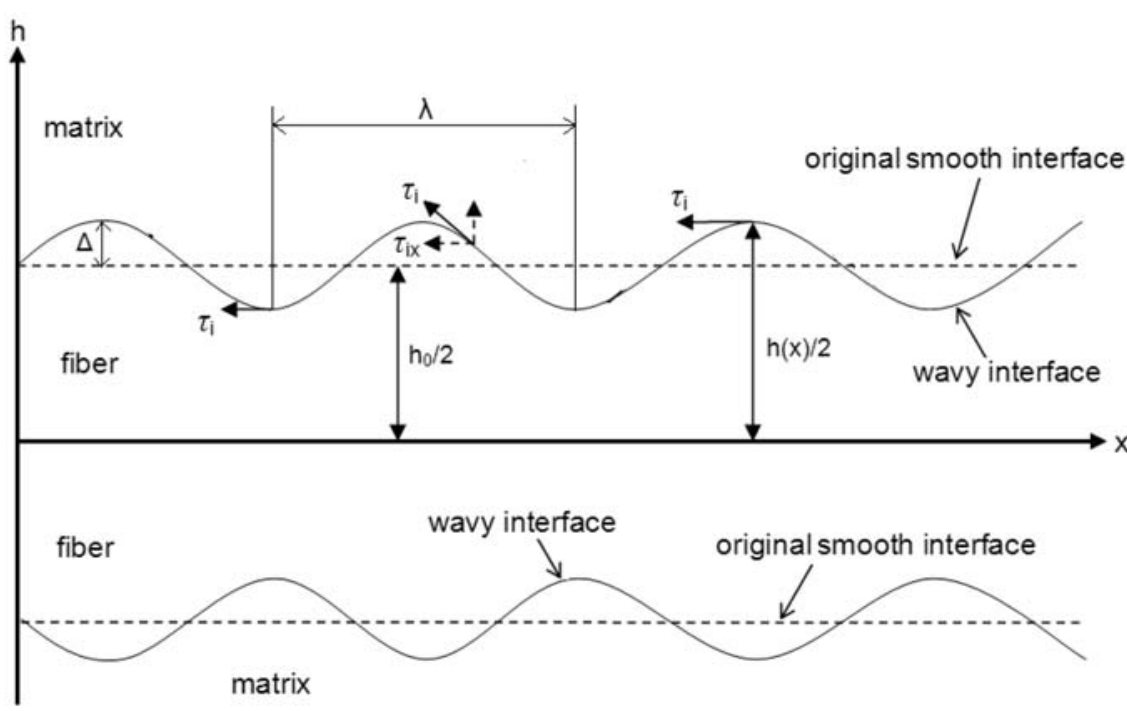

Figure 4. (a) Staggered composites with rough fiber/matrix interfaces and a schematically infinitesimal fiber element for the case of frictional shear transfer and (b) a schematic of the wavy interface and $x$-axis lies on the central line of the fiber.

of each fiber platelet is periodically variable in the length direction, i.e. $h=h(x)$, which is often characterized by a cosine function, ${ }^{18}$

$$
\frac{h(x)}{2}=\frac{h_{0}}{2}+\Delta \cos \left(\frac{2 \pi x}{\lambda}\right)
$$

where $h_{0}$ is the thickness of fibers with a smooth surface as shown in Figure 4(b). With regard to a rough fiber element in length $d x$, the total area of the wavy surface $A_{\tau}$ is:

$$
\begin{aligned}
A_{\tau}(x) & =2 d s \cdot 1=2 \sqrt{\left(\frac{d h}{2}\right)^{2}+(d x)^{2}} \cdot 1 \\
& =2 \sqrt{1+\left(\frac{1}{2} \frac{d h}{d x}\right)^{2}} d x \cdot 1
\end{aligned}
$$

where $d s$ is the length of the boundary of the fiber element. One can see that in equation (4), an enlargement of interfacial contact area by a factor of $\left[1+(d h / 2 d x)^{2}\right]^{1 / 2}$ is induced due to the interface roughness.

In Figure 4(b), $\tau_{i}$ is the interfacial shear stress, which is tangent to the curved interface with $\tau_{i x}$ being the component in $x$ direction. For simplicity, it is assumed that the fiber stress and interfacial shear stress are independent of the fiber's thickness. The cross-sectional area of the fiber is $A(x)=h(x) \cdot 1$ since the thickness $h$ is a function of $x$ due to the curved boundary. Then, the equilibrium equation of the infinitesimal fiber element in $x$ direction can be formulated as

$$
\begin{aligned}
& {\left[\sigma_{f}(x)+d \sigma_{f}(x)\right] \cdot[h(x)+d h(x)]+2 \tau_{i x}(x) .} \\
& \quad d s=\sigma_{f}(x) \cdot h(x)
\end{aligned}
$$


Combining equations (3)-(5) yields

$$
\frac{d\left[\sigma_{f}(x) \cdot h(x)\right]}{d x}=-2 \tau_{i x}(x) \sqrt{1+\frac{4 \pi^{2} \Delta^{2}}{\lambda^{2}} \sin ^{2}\left(\frac{2 \pi x}{\lambda}\right)}
$$

which is the improved shear-lag equation considering the influence of interface roughness.

Compared to the shear-lag equation for the smooth interface model in equation (2), equation (6) characterizes the augmentation of interface area, which is an essential consequence of the surface modification. ${ }^{7,8}$ It is noted that when $\Delta \rightarrow 0$ or $\lambda \rightarrow \infty$, the interface tends to be smooth, which results in $h(x) \rightarrow h_{0}$, $\tau_{i x}=\tau_{i}$. Equation (6) can be naturally reduced to equation (2).

To solve equation (6), it is necessary for us to determine the detailed expression of interfacial shear stress $\tau_{i x}(x)$. Since the polymer matrix can be thermoplastic (PMMA; polycarbonate) or thermosetting (epoxy resin) materials, two kinds of stress transfer forms should be considered, i.e., the frictional shear transfer and the elastic shear one. ${ }^{19,20}$ In the following parts, the above improved shear-lag model will be applied to analyze the two transfer cases, respectively.

\section{Analysis of the frictional shear transfer case}

Frictional shear transfer commonly exists in thermoplastic composites, with the main feature that load transfer at the perfect interface is realized through a constant shear stress which is not affected by Poisson's ratio effects. ${ }^{19,27}$

For a smooth interface, let $\tau_{i}$ in equation (2) be a constant $\tau_{0}$, and the matrix shear stress $\tau_{m}$ equals $\tau_{0}$. It can be derived that $\sigma_{f}$ has a simple linear distribution (the tensile loads at both ends of fiber platelet are neglected as done in literatures), ${ }^{28}$

$$
\sigma_{f}(x)=\left\{\begin{array}{cc}
\frac{2 \tau_{0} x}{h_{0}}, & 0 \leq x \leq \frac{L}{2} \\
\frac{2 \tau_{0}(L-x)}{h_{0}}, & \frac{L}{2} \leq x \leq L
\end{array}\right.
$$

According to equation (7), $\sigma_{f}$ increases almost linearly from both ends of the fiber, and reaches the maximum in the middle, exhibiting a triangular pattern, which is consistent with the results of Kelly-Tyson model. ${ }^{29}$

For a rough interface, the axial tensile load is transferred primarily through $\tau_{i x}$, i.e., $\tau_{i x}$ plays the same role as $\tau_{0}$ does, and equals the matrix shear stress. Hence, it can be considered that the interfacial shear stress in the $x$ direction for the case with a rough interface is independent of the varying thickness, similar to the constant $\tau_{0}$ in the case with a smooth interface. It means that $\tau_{i x}$ distributes uniformly along the rough interface, as $\tau_{0}$ at a smooth interface. Let $\tau_{m}=\tau_{i x}(x)=\tau_{0}$, then equation (6) becomes

$$
\frac{d\left[\sigma_{f}(x) \cdot h(x)\right]}{d x}=-2 \tau_{0} \sqrt{1+\frac{4 \pi^{2} \Delta^{2}}{\lambda^{2}} \sin ^{2}\left(\frac{2 \pi x}{\lambda}\right)}
$$

The right side of equation (8) can also be equivalently interpreted as an increase of the shear stress $\tau_{0}$ acting on smooth interface by a factor of $\sqrt{1+\left(4 \pi^{2} \Delta^{2} / \lambda^{2}\right) \sin ^{2}(2 \pi x / \lambda)}$ due to the interface roughness. Integrating equation (8) from zero to $x$ yields the distribution of $\sigma_{f}$ in the fiber platelet with a rough surface,

$\sigma_{f}(x)= \begin{cases}=\frac{2 \tau_{0} \int_{0}^{x} \sqrt{1+\frac{4 \pi^{2} \Delta^{2}}{\lambda^{2}} \sin ^{2}\left(\frac{2 \pi \xi}{\lambda}\right)} d \xi}{h_{0}+2 \Delta \cos \left(\frac{2 \pi x}{\lambda}\right)}, & 0 \leq x \leq \frac{L}{2} \\ =\frac{2 \tau_{0} \int_{x}^{L} \sqrt{1+\frac{4 \pi^{2} \Delta^{2}}{\lambda^{2}} \sin ^{2}\left(\frac{2 \pi \xi}{\lambda}\right)} d \xi}{h_{0}+2 \Delta \cos \left(\frac{2 \pi x}{\lambda}\right)}, & \frac{L}{2} \leq x \leq L\end{cases}$

Let $\quad \bar{\Delta}=\Delta /\left(h_{0} / 2\right), \quad \bar{\lambda}=\lambda /\left(h_{0} / 2\right), \quad \bar{x}=x /(L / 2)$, $\bar{\xi}=\xi /(L / 2), \bar{h}=h / h_{0}$. The aspect ratio can be denoted as $\rho=L / h \approx L / h_{0}$ due to rather small surface asperities compared to the value of $h_{0}$. Then equation (9) can be rewritten as

$$
\sigma_{f}(\bar{x})= \begin{cases}\frac{\rho \tau_{0} \int_{0}^{\bar{x}} \sqrt{1+\frac{4 \pi^{2} \bar{\Delta}^{2}}{\bar{\lambda}^{2}} \sin ^{2}\left(\frac{2 \pi \rho \bar{\xi}}{\bar{\lambda}}\right)} d \bar{\xi}}{1+\bar{\Delta} \cos \left(\frac{2 \pi \rho \bar{x}}{\bar{\lambda}}\right)}, & 0 \leq \bar{x} \leq 1 \\ \frac{\rho \tau_{0} \int_{\bar{x}}^{2} \sqrt{1+\frac{4 \pi^{2} \bar{\Delta}^{2}}{\bar{\lambda}^{2}} \sin ^{2}\left(\frac{2 \pi \rho \bar{\xi}}{\bar{\lambda}}\right) d \bar{\xi}}}{1+\bar{\Delta} \cos \left(\frac{2 \pi \rho \bar{x}}{\bar{\lambda}}\right)}, & 1 \leq \bar{x} \leq 2\end{cases}
$$

Numerical calculations can find that the value of equation (10) is almost independent of the aspect ratio $\rho$ in the sine and cosine functions. Therefore, equation (10) can be further simplified as

$$
\bar{\sigma}_{f}(\bar{x})=\frac{\sigma_{f}}{\rho \tau_{0}} \approx \begin{cases}\frac{\int_{0}^{\bar{x}} \sqrt{1+\frac{4 \pi^{2} \bar{\Delta}^{2}}{\bar{\lambda}^{2}} \sin ^{2}\left(\frac{2 \pi \bar{\xi}}{\bar{\lambda}}\right)} d \bar{\xi}}{1+\bar{\Delta} \cos \left(\frac{2 \pi \bar{x}}{\bar{\lambda}}\right)}, & 0 \leq \bar{x} \leq 1 \\ \frac{\int_{\bar{x}}^{2} \sqrt{1+\frac{4 \pi^{2} \bar{\Delta}^{2}}{\bar{\lambda}^{2}} \sin ^{2}\left(\frac{2 \pi \bar{\xi}}{\bar{\lambda}}\right)} d \bar{\xi}}{1+\bar{\Delta} \cos \left(\frac{2 \pi \bar{x}}{\bar{\lambda}}\right)}, & 1 \leq \bar{x} \leq 2\end{cases}
$$


Equation (10) gives the normalized fiber stress, which depends only on the parameters related to the surface roughness, $\Delta$ and $\lambda$. Based on equation (11), the average tensile stress in fibers can be found,

$$
\begin{aligned}
<\sigma_{f}>= & \rho \tau_{0}<\bar{\sigma}_{f}> \\
= & \frac{\rho \tau_{0}}{2}\left[\int_{0}^{1}\left(\frac{\int_{0}^{\bar{x}} \sqrt{1+\frac{4 \pi^{2} \bar{\Delta}^{2}}{\bar{\lambda}^{2}} \sin ^{2}\left(\frac{2 \pi \bar{\xi}}{\bar{\lambda}}\right)} d \bar{\xi}}{1+\bar{\Delta} \cos \left(\frac{2 \pi \bar{x}}{\bar{\lambda}}\right)}\right) d \bar{x}\right. \\
& \left.+\int_{1}^{2}\left(\frac{\int_{\bar{x}}^{2} \sqrt{1+\frac{4 \pi^{2} \bar{\Delta}^{2}}{\bar{\lambda}^{2}} \sin ^{2}\left(\frac{2 \pi \bar{\xi}}{\bar{\lambda}}\right)} d \bar{\xi}}{1+\bar{\Delta} \cos \left(\frac{2 \pi \bar{x}}{\bar{\lambda}}\right)}\right) d \bar{x}\right]
\end{aligned}
$$

Since it is assumed that the soft polymer matrix in staggered composites cannot carry the tensile load, the effective tensile stress is

$$
\sigma_{e}=\Phi<\sigma_{f}>
$$

where $\Phi$ represents the volume fraction of fibers in fiber-reinforced polymer composites and $\Phi=h /(h+t) \approx h_{0} /\left(h_{0}+t_{0}\right)$. The effective tensile strain consists of that induced by the shear deformation in matrix and the tensile deformation in fiber platelets. ${ }^{28}$ Since the matrix shear stress $\tau_{m}=\tau_{i x}=\tau_{0}$, we have

$$
\begin{aligned}
\varepsilon_{e}= & \frac{u_{f}+u_{m}}{L}=<\varepsilon_{f}>+\frac{2 \varepsilon_{m}^{\tau} t}{L}=\frac{<\sigma_{f}>}{E_{f}} \\
& +2 \frac{\tau_{0} h(1-\Phi)}{G_{m} L \Phi}
\end{aligned}
$$

where $E_{f}$ and $G_{m}$ denote the Young's modulus of fibers and the shear modulus of matrix. $\varepsilon_{m}^{\tau}$ is the shear strain of matrix. Combining equations (12)-(14) yields the effective Young's modulus of the staggered composite for the case of frictional shear transfer,

$$
E_{e}=\frac{\sigma_{e}}{\varepsilon_{e}}=\frac{\Phi<\sigma_{f}>}{\frac{<\sigma_{f}>}{E_{f}}+\frac{2 \tau_{0} h(1-\Phi)}{G_{m} L \Phi}}=\frac{1}{\frac{1}{\Phi E_{f}}+\frac{2(1-\Phi)}{G_{m} \rho^{2} \Phi^{2}<\bar{\sigma}_{f}>}}
$$

which has a similar form to that in the tensionshear-chain (TSC) model. ${ }^{28}$ From equations (11) and (15), one can find the stress distributions in fibers and effective Young's modulus of a fiberreinforced polymer composite with rough fiber/matrix interfaces.

\section{Analysis of the elastic shear transfer case}

In the thermosetting composites, tensile load is transferred primarily by elastic shear deformation at the interface. ${ }^{19}$ Considering the orthotropic mirror symmetries of the staggered array, the dashed-box as shown in Figure 5 is chosen as a representative unit cell, ${ }^{30,31}$ in which two fiber platelets with half length and half thickness are contained and marked with 1 and 2, respectively. Thermosetting polymer matrix is the material between the two fiber platelets. Due to the waviness of rough interface, the thickness of fiber platelet is relevant to $x$, i.e. $h=h(x)$. The description of the geometry of fiber's rough surface is the same as that in Section An improved shear-lag model. When $h \rightarrow h_{0}$ and $t \rightarrow t_{0}$, the interface tends to be smooth. Moreover, since the longitudinal gap between two fibers is rather small as compared to $L / 2$, the total length of the unit cell is approximately taken as $L / 2$. The deformation pattern of the unit cell and the infinitesimal elements are shown in Figure 6(a) and (b).

In this case, the rough interface between fibers and matrix should be maintained perfect during shear deformation. Under a uniform tension $p$, the fiber stress will reach its maximum value $P_{\max }$ at $x=0$ and $x=L / 2$, which acts as the boundary conditions in the unit cell.

At the rough interface, the tensile stress is transferred via the interfacial shear stress in $x$ direction, i.e., $\tau_{i x}$. Therefore, $\tau_{i x}$ should follow the classical Hooke's law ${ }^{30}$ as

$$
\tau_{i x}(x)=\frac{G_{m}\left[u_{2}(x)-u_{1}(x)\right]}{t}
$$

The shear stress $\tau_{m}$ in the matrix equals $\tau_{i x} . u_{2}(x)$ and $u_{1}(x)$ denote the tensile displacements of fibers 2 and 1 , respectively. Then, the fiber stresses $\sigma_{f 1}$ and $\sigma_{f 2}$ can be written as,

$$
\sigma_{f i}=E_{f} \frac{d u_{i}(x)}{d x}, i=1,2
$$

Similar to Section An improved shear-lag model, the equilibrium equations for fibers 1 and 2 with rough surfaces can be obtained,

fiber 1:

$$
\frac{d\left[\sigma_{f 1}(x) \cdot h(x)\right]}{d x}=-2 \tau_{i x}(x) \sqrt{1+\frac{4 \pi^{2} \Delta^{2}}{\lambda^{2}} \sin ^{2}\left(\frac{2 \pi x}{\lambda}\right)}
$$

fiber 2:

$$
\frac{d\left[\sigma_{f 2}(x) \cdot h(x)\right]}{d x}=2 \tau_{i x}(x) \sqrt{1+\frac{4 \pi^{2} \Delta^{2}}{\lambda^{2}} \sin ^{2}\left(\frac{2 \pi x}{\lambda}\right)}
$$




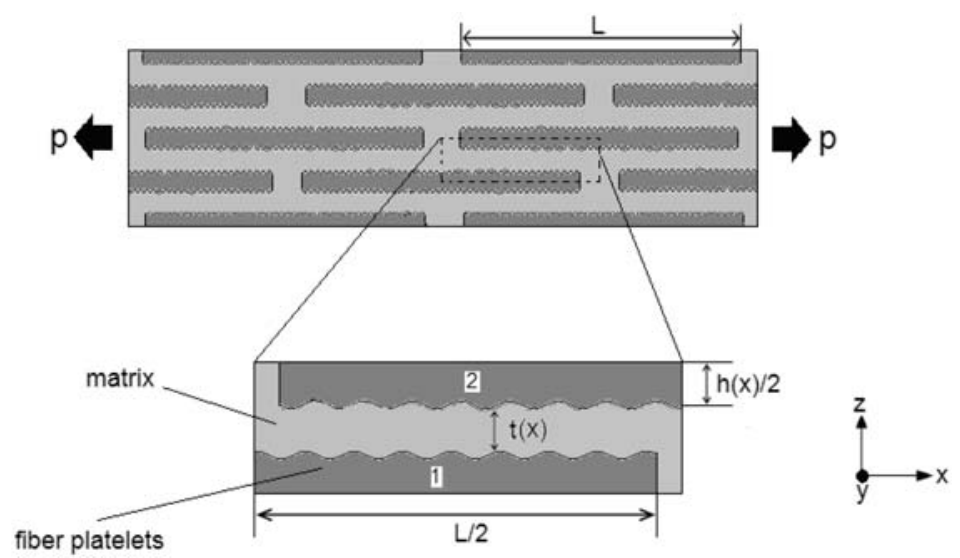

Figure 5. A representative unit cell of staggered composites with rough interface for the case of elastic shear transfer.

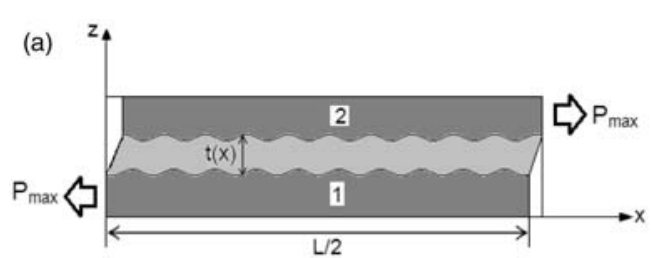

(b)
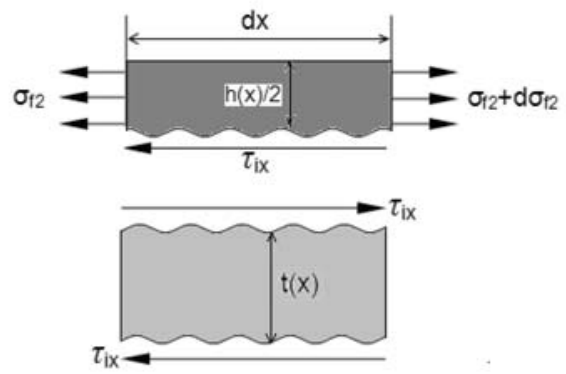

$\tau_{\mathrm{ix}}$

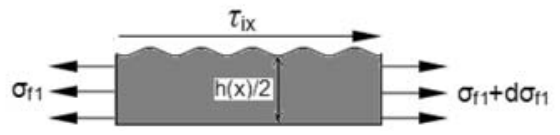

Figure 6. Mechanical behaviors of a unit cell: (a) deformation pattern of unit cell and (b) infinitesimal fiber and matrix elements.

Combining equations (16)-(19) yields:

$$
\left\{\begin{array}{c}
E_{f} \frac{d^{2} u_{1}}{d x^{2}}+\frac{1}{h} \frac{d h}{d x}\left(E_{f} \frac{d u_{1}}{d x}\right)+\frac{2 G_{m}}{t} \frac{u_{2}-u_{1}}{h} \\
\times \sqrt{1+\frac{4 \pi^{2} \Delta^{2}}{\lambda^{2}} \sin ^{2}\left(\frac{2 \pi x}{\lambda}\right)}=0 \\
E_{f} \frac{d^{2} u_{2}}{d x^{2}}+\frac{1}{h} \frac{d h}{d x}\left(E_{f} \frac{d u_{2}}{d x}\right)-\frac{2 G_{m}}{t} \frac{u_{2}-u_{1}}{h} \\
\times \sqrt{1+\frac{4 \pi^{2} \Delta^{2}}{\lambda^{2}} \sin ^{2}\left(\frac{2 \pi x}{\lambda}\right)}=0
\end{array}\right.
$$

Using the non-dimensional parameters $\bar{\Delta}=$ $\Delta /\left(h_{0} / 2\right), \quad \bar{\lambda}=\lambda /\left(h_{0} / 2\right), \quad \bar{h}=h / h_{0}, \quad \bar{x}=x /(L / 2)$, $\bar{u}_{i}=u_{i} /(L / 2),(i=1,2)$, and the aspect ratio $\rho \approx L / h_{0}$, volume fraction of fibers $\Phi \approx h_{0} /\left(h_{0}+t_{0}\right)$, Equation (20) can be rewritten as

$$
\left\{\begin{array}{l}
E_{f} \frac{d^{2} \bar{u}_{1}}{d \bar{x}^{2}}+\frac{\rho}{2} \cdot \frac{1}{\bar{h}} \frac{d h}{d x}\left(E_{f} \frac{d \bar{u}_{1}}{d \bar{x}}\right)+\frac{\rho^{2}}{2} \cdot \frac{G_{m} \Phi}{1-\Phi} \frac{\bar{u}_{2}-\bar{u}_{1}}{\bar{h}^{2}} \\
\times \sqrt{1+\frac{4 \pi^{2} \bar{\Delta}^{2}}{\bar{\lambda}^{2}} \sin ^{2}\left(\frac{2 \pi \rho \bar{x}}{\bar{\lambda}}\right)}=0 \\
E_{f} \frac{d^{2} \bar{u}_{2}}{d \bar{x}^{2}}+\frac{\rho}{2} \cdot \frac{1}{\bar{h}} \frac{d h}{d x}\left(E_{f} \frac{d \bar{u}_{2}}{d \bar{x}}\right)-\frac{\rho^{2}}{2} \cdot \frac{G_{m} \Phi}{1-\Phi} \frac{\bar{u}_{2}-\bar{u}_{1}}{\bar{h}^{2}} \\
\times \sqrt{1+\frac{4 \pi^{2} \bar{\Delta}^{2}}{\bar{\lambda}^{2}} \sin ^{2}\left(\frac{2 \pi \rho \bar{x}}{\bar{\lambda}}\right)}=0
\end{array}\right.
$$

where $\bar{h}=1+\bar{\Delta} \cos (2 \pi \rho \bar{x} / \bar{\lambda})$ and $d h / d x=-(4 \pi \bar{\Delta} / \bar{\lambda})$ $\sin (2 \pi \rho \bar{x} / \bar{\lambda})$.

Considering the boundary conditions of the unit cell, the fiber stresses $\sigma_{f 1}$ and $\sigma_{f 2}$ can be normalized as

$$
\bar{\sigma}_{f i}=\frac{\sigma_{f i}}{P_{\max }}=\frac{E_{f} \frac{d u_{i}}{d x}}{P_{\max }}=\frac{E_{f} \frac{d \bar{u}_{i}}{d \bar{x}}}{P_{\max }}=\frac{d}{d \bar{x}}\left(\frac{E_{f} \bar{u}_{i}}{P_{\max }}\right), i=1,2
$$

Let $f_{1}(\bar{x})=E_{f} \bar{u}_{1}(\bar{x}) / P_{\max }, f_{2}(\bar{x})=E_{f} \bar{u}_{2}(\bar{x}) / P_{\max }$, then equation (21) becomes

$$
\left\{\begin{array}{c}
\frac{d^{2} f_{1}}{d \bar{x}^{2}}+\frac{\rho}{2} \cdot \frac{1}{\bar{h}} \frac{d h}{d x} \cdot \frac{d f_{1}}{d \bar{x}}+\frac{\rho^{2} \kappa^{2}}{2} \frac{f_{2}-f_{1}}{\bar{h}} \\
\times \sqrt{1+\frac{4 \pi^{2} \bar{\Delta}^{2}}{\bar{\lambda}^{2}} \sin ^{2}\left(\frac{2 \pi \rho \bar{x}}{\bar{\lambda}}\right)}=0 \\
\frac{d^{2} f_{2}}{d \bar{x}^{2}}+\frac{\rho}{2} \cdot \frac{1}{\bar{h}} \frac{d h}{d x} \cdot \frac{d f_{2}}{d \bar{x}}-\frac{\rho^{2} \kappa^{2}}{2} \frac{f_{2}-f_{1}}{\bar{h}} \\
\times \sqrt{1+\frac{4 \pi^{2} \bar{\Delta}^{2}}{\bar{\lambda}^{2}} \sin ^{2}\left(\frac{2 \pi \rho \bar{x}}{\bar{\lambda}}\right)}=0
\end{array}\right.
$$


where $\kappa=\left[G_{m} \Phi / E_{f}(1-\Phi)\right]^{1 / 2}, f_{1}=f_{1}(\bar{x}), f_{2}=f_{2}(\bar{x})$. One can see that equation (23) contains two 2-order ordinary differential equations with respect to $f_{1}$ and $f_{2}$.

The boundary conditions are

$$
\begin{aligned}
& \text { At } \bar{x}=0 \text { (left end) }: f_{1}=0, \frac{d f_{1}}{d \bar{x}}=1, \frac{d f_{2}}{d \bar{x}}=0 \\
& \text { At } \bar{x}=1 \text { (right end) }: f_{2}=0, \frac{d f_{2}}{d \bar{x}}=1, \frac{d f_{1}}{d \bar{x}}=0
\end{aligned}
$$

Moreover, using the definition of $f_{1}$ and $f_{2}$, equation (16) can be re-formulated as

$$
\tau_{i x}(x)=\frac{\rho \kappa^{2} P_{\max }\left(f_{2}-f_{1}\right)}{2}
$$

where the relations $\rho \approx L / h_{0}$ and $\Phi \approx h_{0} /\left(h_{0}+t_{0}\right)$ have been used.

Since the unit cell contains two fibers with the same size, the effective tensile stress is

$$
\begin{aligned}
\sigma_{e} & =\frac{\Phi}{2}\left[<\sigma_{f 1}>+<\sigma_{f 2}>\right] \\
& =\frac{\Phi}{2}\left[\frac{2}{L}\left(\int_{0}^{L / 2} \sigma_{f 1}(x) d x+\int_{0}^{L / 2} \sigma_{f 2}(x) d x\right)\right] \\
& =\frac{\Phi P_{\max }}{2}\left(\int_{0}^{1} \bar{\sigma}_{f 1}(\bar{x}) d \bar{x}+\int_{0}^{1} \bar{\sigma}_{f 2}(\bar{x}) d \bar{x}\right)
\end{aligned}
$$

According to Zuo and $\mathrm{Wei},{ }^{30}$ the overall strain of the unit cell as shown in Figure 6(a) is

$\varepsilon_{e}=<\varepsilon_{f 1}>+\frac{\tau_{m}(0) t}{G_{m} \cdot(L / 2)}=\frac{<\sigma_{f 1}>}{E_{f}}+\frac{2\left[u_{2}(0)-u_{1}(0)\right]}{L}$

Combining equations (22) and (26), equation (28) becomes:

$$
\begin{aligned}
\varepsilon_{e} & =\frac{<\sigma_{f 1}>}{E_{f}}+\frac{2\left[u_{2}(0)-u_{1}(0)\right]}{L} \\
& =\frac{P_{\max }}{E_{f}}\left[\int_{0}^{1} \bar{\sigma}_{f 1}(\bar{x}) d \bar{x}+f_{2}(0)-f_{1}(0)\right]
\end{aligned}
$$

Then, the effective Young's modulus of fiber-reinforced composites can be written as

$$
E_{e}=\frac{\sigma_{e}}{\varepsilon_{e}}=\frac{\Phi E_{f}\left(<\bar{\sigma}_{f 1}>+<\bar{\sigma}_{f 2}>\right)}{2\left[<\bar{\sigma}_{f 1}>+f_{2}(0)-f_{1}(0)\right]}
$$

Based on equations (17), (22)-(26) and (30), the tensile stresses in fibers 1 and 2, the shear stress in thermosetting matrix and the overall Young's modulus of composites can be found. In the following section, the effects of interface roughness on these terms will be analyzed for the two kinds of stress-transfer forms, respectively.

\section{Results and discussions}

Consider a unidirectional staggered composite system with stiff fibers and soft polymer matrix. The material parameters are taken as $E_{f}=200 \mathrm{GPa}, E_{m}=4 \mathrm{GPa}$, $v_{f}=0.2, v_{m}=0.3$ and the volume fraction $\Phi=0.45$, where the subscripts $f$ and $m$ represent the fiber and matrix, respectively. The surface roughness is described by the ratio of the amplitude to the wavelength, i.e., $\bar{\Delta} / \bar{\lambda}$. When the ratio approaches zero, the fiber/ matrix interface tends to be smooth; and when the ratio increases, the interface will become much rougher. According to the former researches, ${ }^{10,16}$ for a rough interface, the dimensionless amplitude $\bar{\Delta}$ is assumed to be 0.001 , i.e. nano-sized asperities.

\section{The frictional shear transfer case}

According to equation (11), distributions of the tensile stress in the fiber platelet along the fiber length for different interface roughness are given in Figure 7. Results for the case with a smooth interface are also plotted for a comparison. Not only the case with a smooth interface but also the cases with different interface roughness exhibit a similar triangular shape, but the fiber stress increases significantly when the ratio of $\bar{\Delta} / \bar{\lambda}$ increases. It means that in the frictional shear transfer case, the interface roughness will enable fibers to carry more tensile loads (assuming the fiber stress lying within the range of its theoretical tensile strength). This phenomenon may be explained by the lack of chemical functional groups in the thermoplastic matrix, and the interfacial adhesion stems primarily from fiber-matrix mechanical interlocking. ${ }^{19}$ As interface roughness increases, the real contact area between fiber and matrix is enlarged, leading to an enhancement of mechanical interlocking. Therefore, higher tensile stress can be transferred via the rough interface from matrix to fibers. Since fibers are generally much stiffer than soft organic matrix, the overall load-bearing capacity of composites can be effectively optimized in this case, which is just one of the main aims that designers expect to achieve through surface modification.

Figure 8 gives the relation between the effective Young's modulus of staggered composites and the aspect ratio of fibers under different interface roughness. Similar to the results in TSC model, ${ }^{28}$ the effective Young's modulus $E_{e}$ rises rapidly with the increase of aspect ratio $\rho$, and gradually approaches a constant value as $\rho$ is large enough $(\geq 100)$. With regard to the 


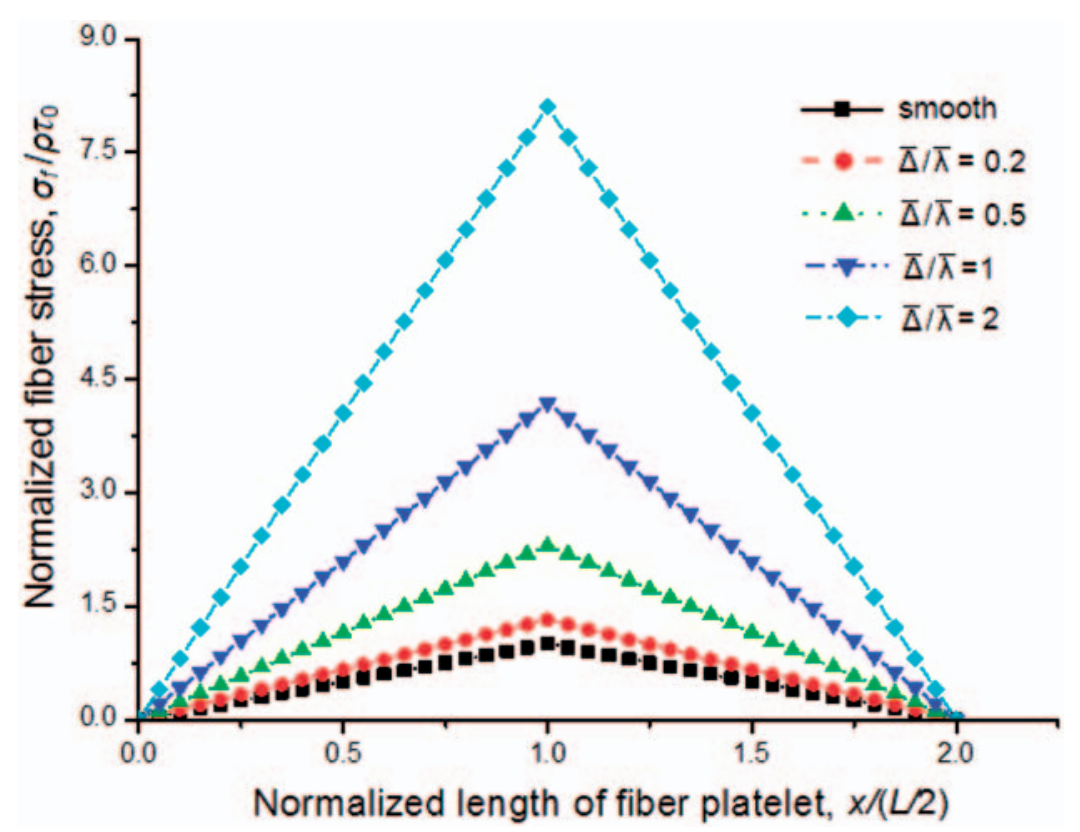

Figure 7. Distributions of fiber stresses along the fiber length with different interface roughness.

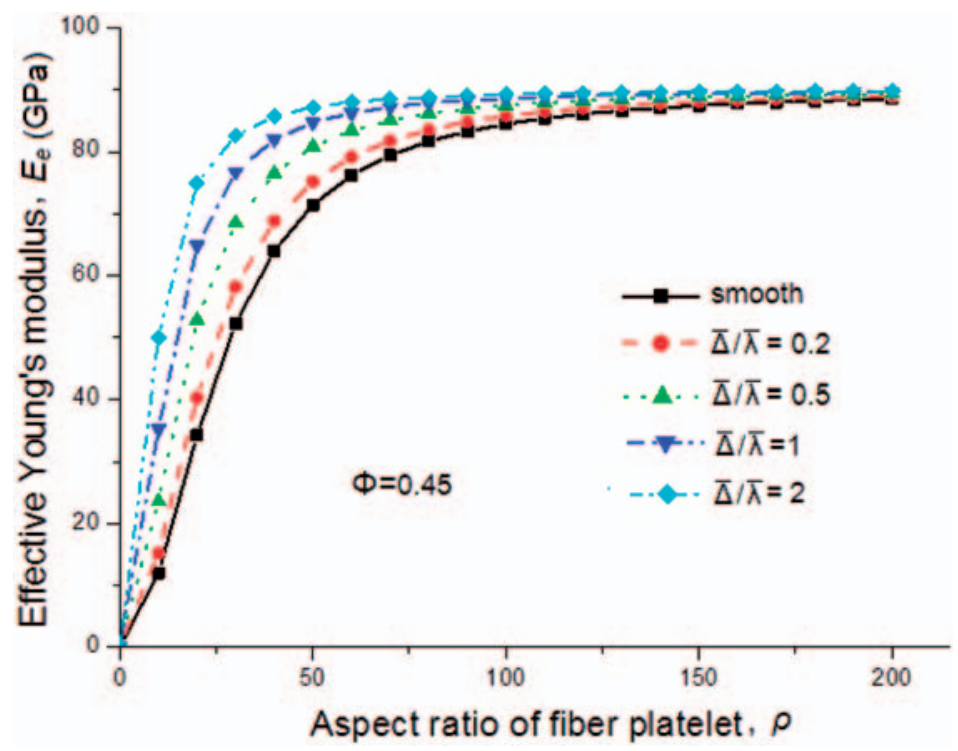

Figure 8. The effective Young's modulus of composites versus the aspect ratio of fibers for the case of frictional shear transfer with $\Phi=0.45$ and different interface roughness.

effect of interface roughness, it shows that the larger the ratio $\bar{\Delta} / \bar{\lambda}$, the higher the effective Young's modulus $E_{e}$ will be, which reflects an enhancing effect of interface roughness on the stiffness of composites. However, $E_{e}$ increases very sharply at the initial stage with the increasing $\bar{\Delta} / \bar{\lambda}$ and then tends to a constant that seems insensitive to the larger aspect ratio or roughness.

Similar trends are also found for the relation between the effective Young's modulus and the volume fraction of fibers as shown in Figure 9. One can see that in the case with a small aspect ratio as shown in Figure 9(a), the effective Young's modulus increases with an increasing volume fraction of fibers when the interface roughness keeps unchanged. For a determined volume fraction of fibers, the effective Young's modulus also increases significantly with an increasing interface roughness. However, comparing Figure 9(a)-(c), in which the aspect ratio of fibers 

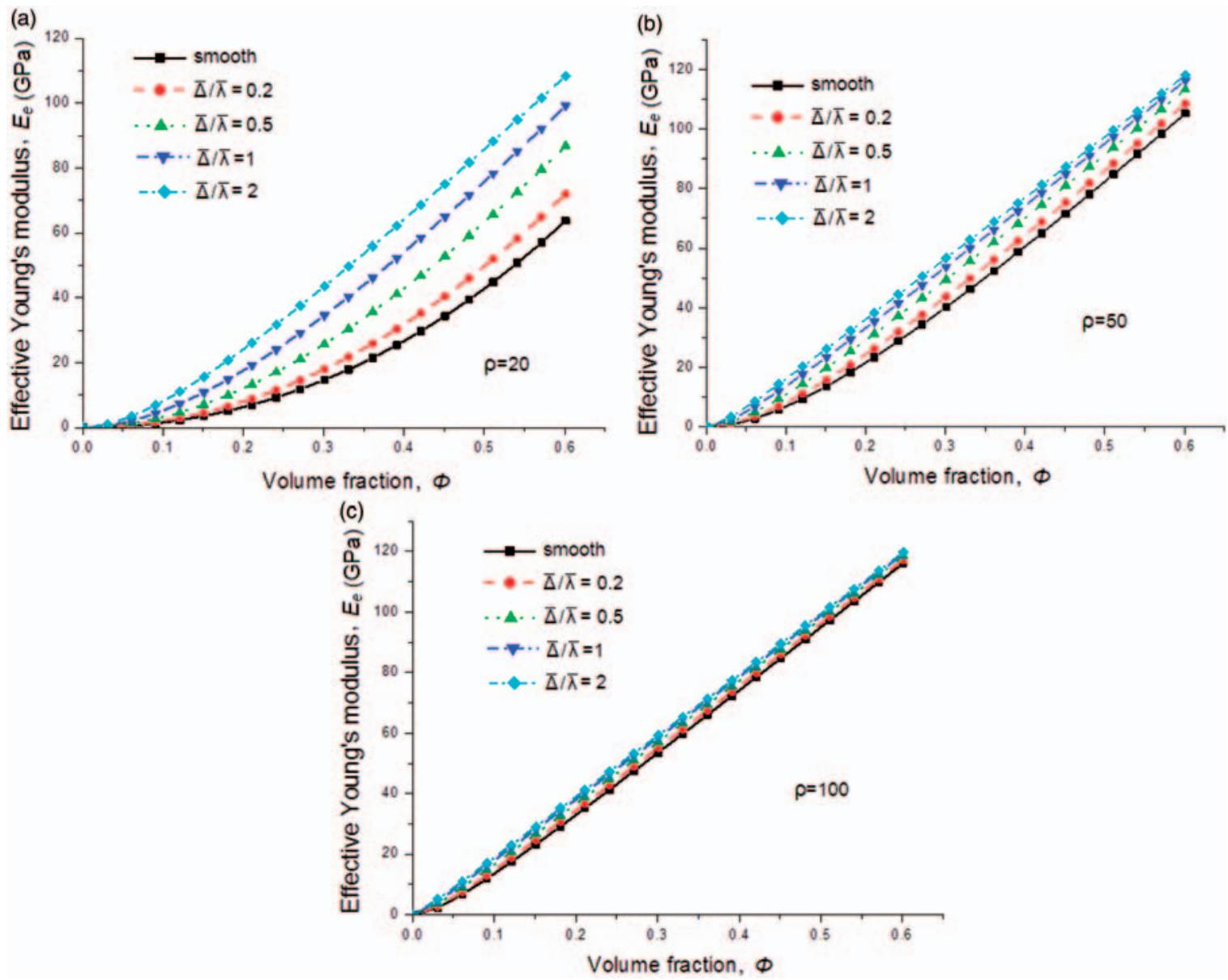

Figure 9. The effect of interface roughness on the effective Young's modulus as a function of the volume fraction of fibers for the case of frictional shear transfer with different aspect ratios: (a) for $\rho=20$, (b) for $\rho=50$ and (c) for $\rho=100$.

increases from 20 to 100 , the effect of the interface roughness on the effective Young's modulus reduces obviously with an increasing aspect ratio of fibers. Thus, an interesting conclusion could be made that the enhancing effect of interface roughness on the effective Young's modulus $E_{e}$ is only significant for fibers with a small and medium aspect ratio; for the case with a large aspect ratio of fibers, the effect of fiber/matrix interface roughness on the stiffness of composites is very weak.

\section{The elastic shear transfer case}

According to equation (26), the non-dimensional shear stress in the thermosetting matrix can be rewritten as

$$
\bar{\tau}_{m}=\bar{\tau}_{i x}=\frac{2 \tau_{i x}}{P_{\max }}=\frac{2 \tau_{m}}{P_{\max }}=\rho \kappa^{2}\left[f_{2}(\bar{x})-f_{1}(\bar{x})\right]
$$

in which $\kappa=\left[G_{m} \Phi / E_{f}(1-\Phi)\right]^{1 / 2}$. From equation (31), we find that the non-dimensional matrix shear stress only depends on the difference of functions $f_{1}$ and $f_{2}$ for given material parameters $\rho, \Phi, E_{f}$ and $G_{m}$. Let the aspect ratio $\rho=30$, solving equation (23) numerically yields the influences of the interface roughness on the distributions of matrix shear stress and the tensile stress in fibers 1 and 2 in the unit cell, which are shown in Figures 10 and 11, respectively.

In Figure 10, one can see that the matrix shear stress distributes parabolically in $x$ direction and reaches the maximum at both ends of the unit cell, exhibiting a similar pattern to the results in References [30,31]. The interface roughness shows significant influence on the matrix shear stress, which decreases with an increasing interface roughness $\bar{\Delta} / \bar{\lambda}$. It means that, in the case of elastic shear transfer, the increase of interface roughness could help to reduce the shear stress in the thermosetting matrix so that matrix fracture is less likely to happen. Moreover, since the matrix shear stress equals the interfacial shear stress, the reduction of $\tau_{i x}$ at $x=0$ and $x=L / 2$ leads to a weakened stress concentration at the fiber/matrix interface. Consequently, a larger external force yielding interface debonding could 


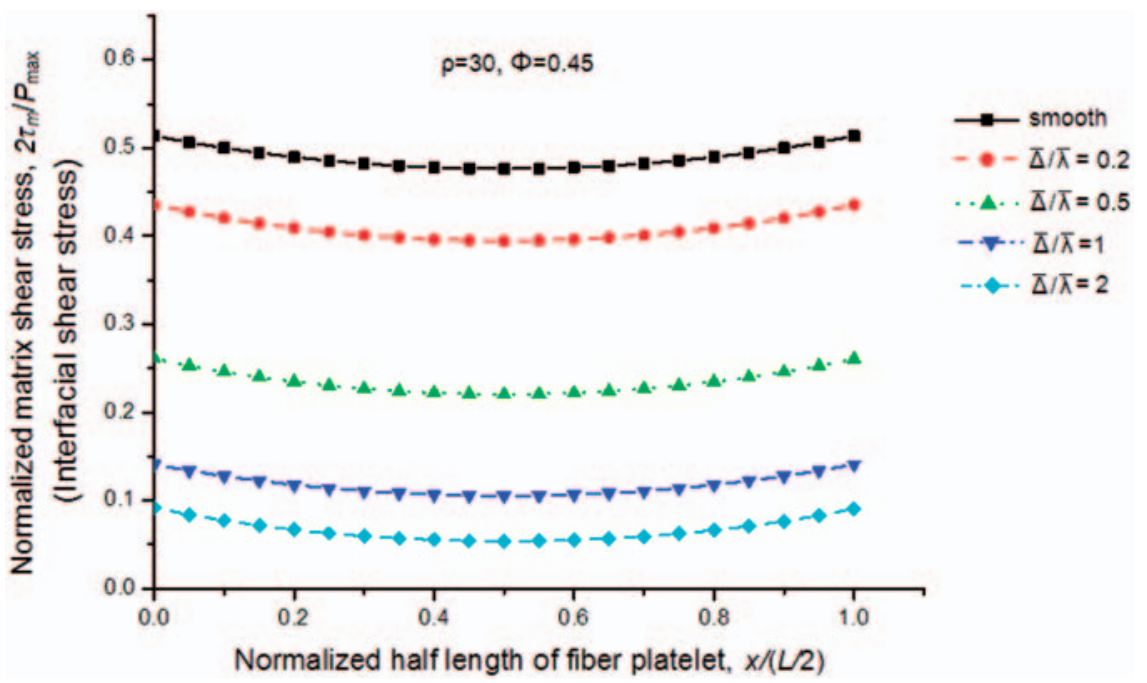

Figure 10. Distributions of the matrix shear stress or the interfacial one for the case of elastic shear transfer with $\rho=30, \Phi=0.45$ and different interface roughness.
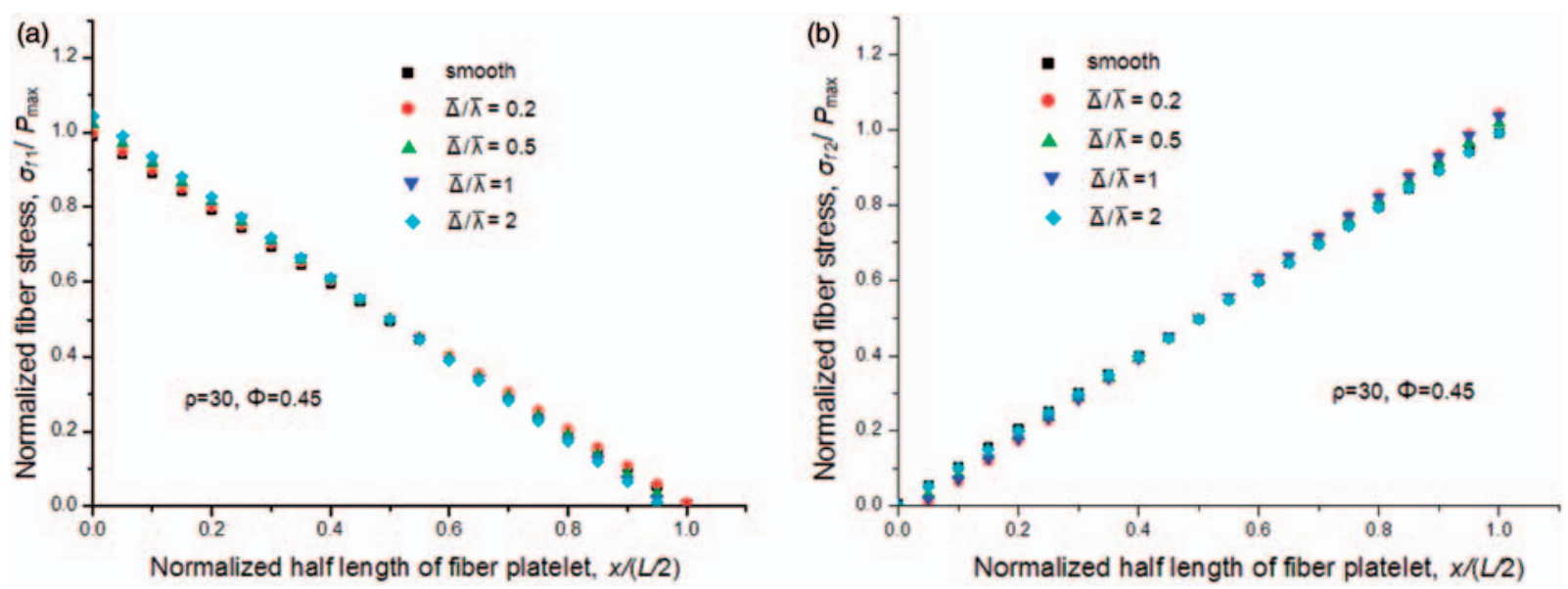

Figure II. Distributions of the tensile stresses in fibers I and 2 for the case of elastic shear transfer with $\rho=30, \Phi=0.45$ and different interface roughness: (a) for $\sigma_{f 1}$ and (b) for $\sigma_{f 2}$.

be supported in the case with a rougher interface. This conclusion agrees well with many experimental observations of surface modification. ${ }^{8-11}$

Figure 11 exhibits the distributions of tensile stress in fibers 1 and 2 with respect to the normalized fiber length $\bar{x}$. Comparing to the case of frictional shear transfer, the interface roughness has hardly influences on the fiber stress in the case of elastic shear transfer. This phenomenon is consistent well with the analytical results of Chai and Mai, ${ }^{16}$ in which the effect of interface roughness on fiber stress is very weak when the fiber/epoxy matrix interface is perfect and subjected to an elastic shear stress. Combining Figures 10 and 11 yields a conclusion that a lower interfacial shear stress is involved to transfer the same amount of tensile loads in the case with a rougher interface. The efficiency of stress-transfer is improved with increasing interface roughness.

The effective Young's modulus is expressed by equation (30), in which the normalized average fiber stress can be obtained from Figure 11,

$$
<\bar{\sigma}_{f 1}>\approx<\bar{\sigma}_{f 2}>\approx 0.5
$$

which almost keep invariants with a varying interface roughness $\bar{\Delta} / \bar{\lambda}$. Thus, equation (30) can be further rewritten as

$$
E_{e}=\frac{\Phi E_{f}\left(<\bar{\sigma}_{f 1}>+<\bar{\sigma}_{f 2}>\right)}{2\left[<\bar{\sigma}_{f 1}>+f_{2}(0)-f_{1}(0)\right]} \approx \frac{\Phi E_{f}}{1+2\left[f_{2}(0)-f_{1}(0)\right]}
$$




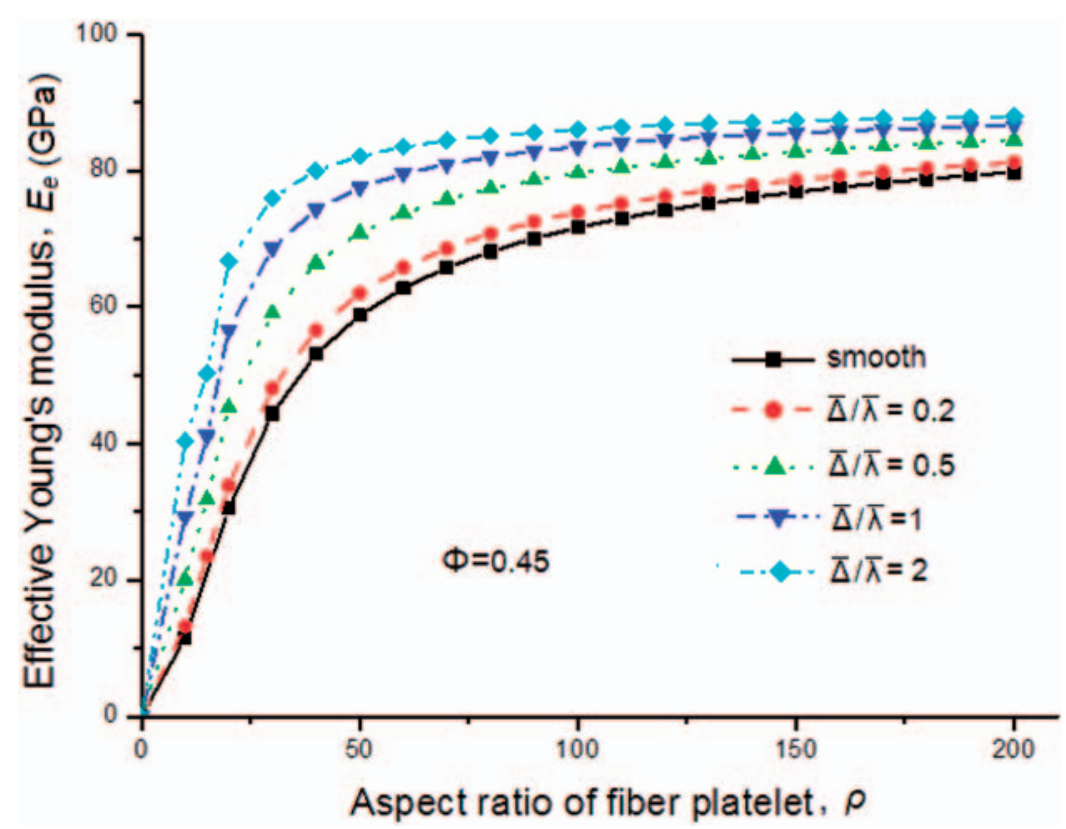

Figure 12. The effective Young's modulus of composites versus the aspect ratio of fibers for the case of elastic shear transfer with $\Phi=0.45$ and different interface roughness.

in which the value of $\left[f_{2}(0)-f_{1}(0)\right]$ for different aspect ratios can be calculated in equation (23). Then, the relation between the effective Young's modulus of composites and the aspect ratio of fibers can be found as shown in Figure 12. The relation between the effective Young's modulus and the volume fraction of fibers are also given in Figure 13 for a fixed aspect ratio of fibers.

Comparing the cases of frictional shear transfer and elastic one as shown in Figures 8 and 9 and Figures 12 and 13 , respectively, we find that the varying trends of effective Young's modulus $E_{e}$ versus the aspect ratio and the volume fraction of fibers are similar in both cases: the effective Young's modulus increases with an increasing volume fraction of fibers when the interface roughness keeps unchanged; for a determined volume fraction of fibers, the effective Young's modulus also increases significantly with an increasing interface roughness. However, the effect of the interface roughness on the effective Young's modulus reduces obviously for cases with a relatively large aspect ratio of fibers. The difference between the two cases is that the range of aspect ratio, within which the interface roughness has a relatively pronounced effect on $E_{e}$, appears to be larger $(\rho \leq 100)$ in the elastic shear transfer case as shown in Figures 12 and 13 than that in the frictional one as shown in Figures 8 and $9(\rho \leq 60)$. This difference is naturally induced by the different enhancing mechanisms of $E_{e}$ in both cases: one is realized through the increase of fiber tensile stress and the other is via the reduction of matrix shear stress.

The function of interface roughness on the overall Young's modulus of polymer composites can be qualitatively predicted based on the new shear-lag model as shown above. The improvement of $E_{e}$ due to roughness seems not large in both stress-transfer cases, which is qualitatively consistent with the phenomenon revealed by Figure 1(b). However, it is still impossible for us to make one-to-one comparison between the analytical and the experimental results due to the lack of detailed experimental data. Further experimental explorations are still needed in the future.

\section{Comparisons between our model and Zhao and Weng}

In Zhao and Weng, ${ }^{32}$ a ribbon-fiber reinforced composite system was investigated, in which the effects of the orientation and volume fraction of ribbon-fibers were considered while the length was assumed to be infinite. For a composite reinforced with monotonically aligned ribbons, its longitudinal effective Young's modulus was formulated as a function of the ribbon fiber's volume fraction $c_{f},{ }^{32}$

$$
\begin{aligned}
& E_{e}=c_{f} E_{f}+\left(1-c_{f}\right) E_{m}+\frac{4 c_{f}\left(1-c_{f}\right)\left(v_{f}-v_{m}\right)^{2}}{c_{f}\left(\frac{1}{\kappa_{m}}+\frac{1}{G_{m}}\right)+\left(1-c_{f}\right)\left(\frac{1}{\kappa_{f}}+\frac{1}{G_{f}}\right)} \\
& \kappa_{f}=\frac{G_{f}}{1-2 v_{f}}, \kappa_{m}=\frac{G_{m}}{1-2 v_{m}}
\end{aligned}
$$

where $E_{f}, G_{f}, \kappa_{f}, v_{f}$ and $E_{m}, G_{m}, \kappa_{m}, v_{m}$ represent the Young's moduli, shear moduli, bulk moduli and 

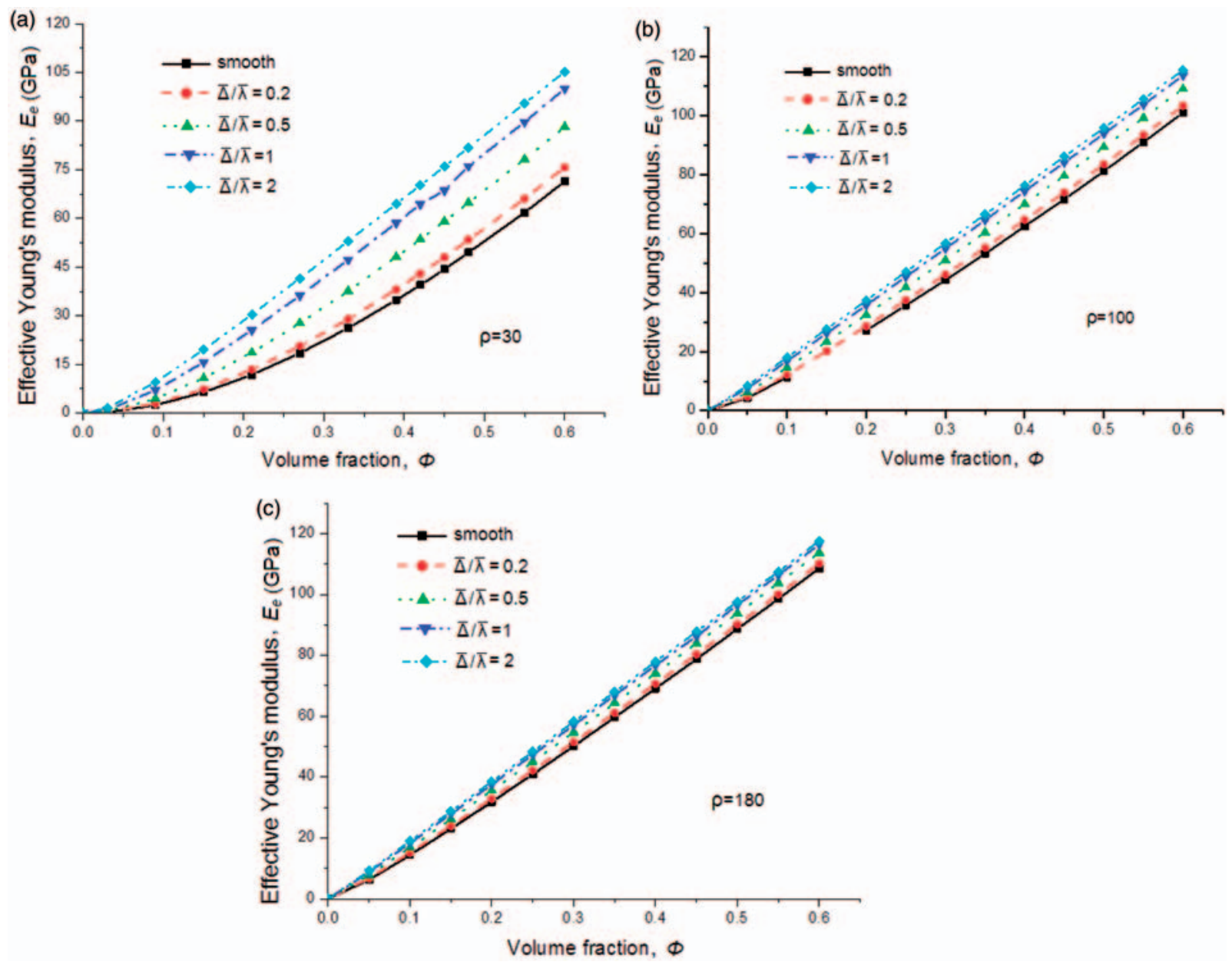

Figure 13. The effect of interface roughness on the effective Young's modulus as a function of volume fraction of fibers for the case of elastic shear transfer with under different aspect ratios: (a) for $\rho=20$, (b) for $\rho=50$ and (c) for $\rho=100$.
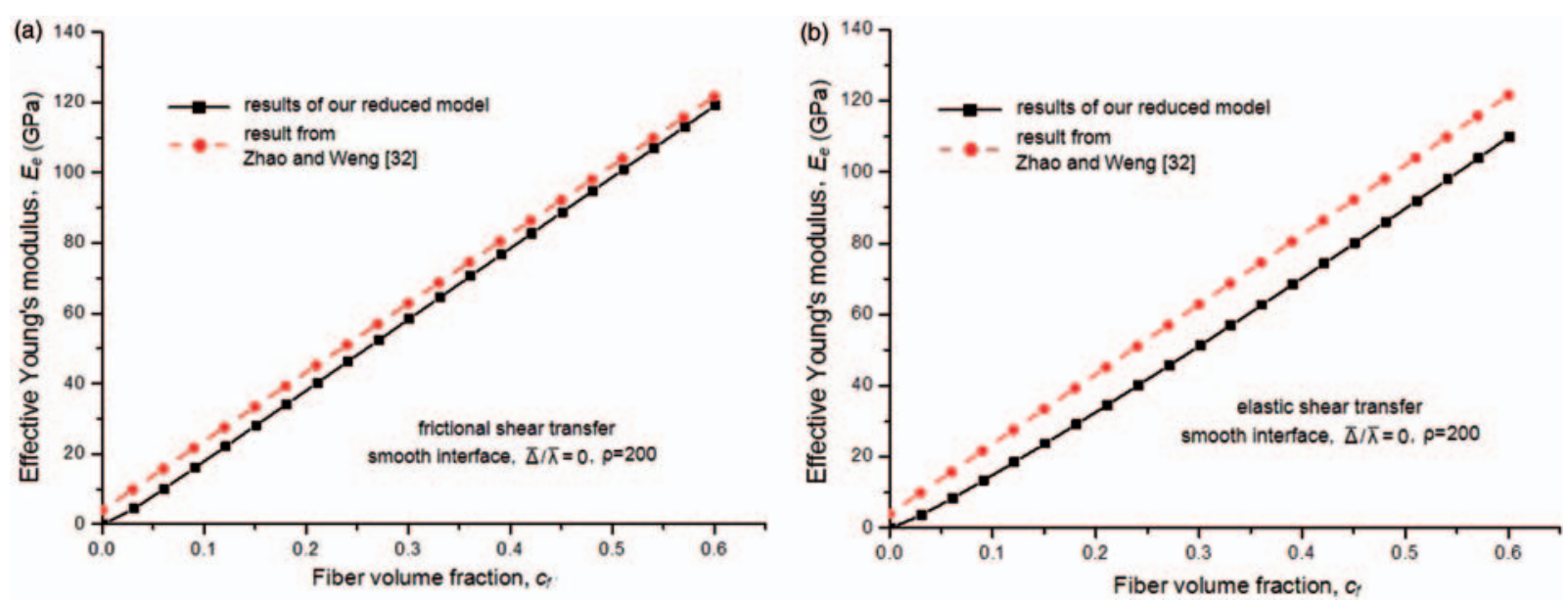

Figure 14. Comparison of the longitudinal effective Young's modulus predicted by Zhao and Weng ${ }^{32}$ and that predicted by our reduced model with an aspect ratio $\rho=200$ : (a) for the frictional shear transfer case and (b) for the elastic shear transfer case. 
Poisson's ratios of the isotropic fiber and matrix, respectively.

In order to compare our results to those obtained by Zhao and Weng, ${ }^{32}$ we reduce our model to the one with a smooth fiber/matrix interface. The calculating longitudinal effective Young's moduli for the frictional shear transfer case and the elastic one are shown and compared to the predictions by Zhao and Weng ${ }^{32}$ as shown in Figure 14(a) and (b), respectively, in which the aspect ratio takes a very large value $(\rho=200)$ due to the assumed infinite fiber length in Reference [32]. It is interesting to find that both the present reduced model and that in Reference [32] give a similar prediction for the longitudinal effective Young's modulus.

\section{Conclusions}

An improved shear-lag model is proposed in order to investigate the effects of interface roughness on the mechanical properties of fiber-reinforced polymer composites. Two different transfer forms of interface stresses are considered, i.e., frictional shear transfer and elastic shear one, which correspond to the cases of choosing thermoplastic and thermosetting polymer materials as the matrix in composites, respectively. In the former, the interfacial shear stress is a constant, and it is found that an increasing interface roughness leads to a significant increase of fiber stresses. That means fibers with a rough surface will sustain more tensile loads than those with a smooth interface. In the latter, it is found that the interface roughness will reduce the shear stresses in thermosetting matrix and at the interface, resulting in an improved strength of composites and the fiber stress keeps invariant for varying interface roughness, which agrees well with the results in Chai and Mai. ${ }^{16}$ Therefore, the influence mechanisms of fiber's surface roughness on stress transfer behaviors in thermoplastic and thermosetting composites are totally different. In both cases, interface roughness and the volume fraction of fibers could enhance the effective Young's modulus of composites. However, the effect of the interface roughness on the effective Young's modulus reduces obviously for cases with a relative large aspect ratio of fibers. All the theoretical results in the present paper should be helpful for the optimum design of interfaces in composites with surface-inert advanced fibers and organic polymer matrix, such as carbon or aramid-reinforced polymer composites.

\section{Funding}

This research received specific grant from the National Natural Science Foundation of China (NSFC).

\section{Acknowledgments}

The work reported here is supported by NSFC through Grants \#10972220, \#11125211, \#11021262 and the 973 Nano-project (2012CB937500).

\section{Conflict of interest}

None declared.

\section{References}

1. Hughes JDH. The carbon fiber/epoxy interface-a review. Compos Sci Technol 1991; 41(1): 31-45.

2. Atkinson KE and Kiely C. The influence of fiber surface properties on the mode of failure in carbon-fiber/epoxy composites. Compos Sci Technol 1998; 58(12): 1917-1922.

3. Moon SI and Jang J. The mechanical interlocking and wetting at the interface between argon plasma treated UHMPE fiber and vinylester resin. J Mater Sci 1999; 34(17): 4219-4224.

4. Chen $\mathrm{P}$, Wang $\mathrm{J}$ and $\mathrm{Li} \mathrm{H}$. Improvement of interfacial adhesion for plasma-treated aramid fibers reinforced poly (phthalazinone ether sulfone ketone) composite and fiber surface aging effects. Surf Interface Anal 2009; 41(1): 38-43.

5. Zhang YH, Huang YD, Liu L, et al. Surface modifications of aramid fibers with $\gamma$-ray radiation for improving interfacial bonding strength with epoxy resin. J Appl Polym Sci 2007; 106(4): 2251-2262.

6. Bai YP, Wang $Z$ and Feng LQ. Interface properties of carbon fiber/epoxy resin composite improved by supercritical water and oxygen in supercritical water. Mater Design 2010; 31(3): 1613-1616.

7. Zhang CS, Chen P, Sun BL, et al. Surface analysis of oxygen plasma treated poly(p-phenylene benzobisoxazole) fibers. Appl Surf Sci 2008; 254(18): 5776-5780.

8. Jang $\mathbf{J}$ and Yang HJ. The effect of surface treatment on the performance improvement of carbon fiber/polybenzoxazine composites. J Mater Sci 2000; 35(9): 2297-2303.

9. Kim SY, Baek SJ and Youn JR. New hybrid method for simultaneous improvement of tensile and impact properties of carbon fiber reinforced composites. Carbon 2011; 49(15): 5329-5338.

10. Song W, Gu AJ, Liang GZ, et al. Effects of surface roughness on interfacial properties of carbon fibers reinforced epoxy resin composites. Appl Surf Sci 2011; 257(9): 4069-4074.

11. Xie JF, Xin DW, Cao HY, et al. Improving carbon fiber adhesion to polyimide with atmospheric pressure plasma treatment. Surf Coat Technol 2011; 206(2-3): 191-201.

12. Kim SN, McMahon PE, Riggs JP, et al. Process for the surface modification of carbon fibers. U.S. Patent 4,374,114, 1983.

13. Sanadi AR and Piggott MR. Interfacial effects in carbonepoxies-Part 1: strength and modulus with short aligned fibres. J Mater Sci 1985; 20(2): 421-430.

14. Rjafiallah S, Guessasma S and Bizot H. Effect of surface etching on interphase and elastic properties of a biocomposite reinforced using glass-silica particles. Compos Sci Technol 2010; 70(8): 1272-1279. 
15. Liu HY, Zhou LM and Mai YW. On fiber pull-out with a rough interface. Philos Mag A 1994; 70(2): 359-372.

16. Chai YS and Mai YW. New analysis on the fiber pushout problem with interface roughness and thermal residual stresses. J Mater Sci 2001; 36(8): 2095-2104.

17. Brandstetter J, Kromp K, Peterlik H, et al. Effect of surface roughness on friction in fiber-bundle pull-out tests. Compos Sci Technol 2005; 65(6): 981-988.

18. Jiang LY, Huang Y, Jiang H, et al. A cohesive law for carbon nanotube/polymer interfaces based on the van der Waals force. J Mech Phys Solids 2006; 54(11): 2436-2452.

19. Huang YL and Young RJ. Interfacial micromechanics in thermoplastic and thermosetting matrix carbon fiber composites. Compos Pt A 1996; 27(10): 973-980.

20. Leveque D and Auvray MH. Study of carbon-fiber strain in model composites by Raman spectroscopy. Compos Sci Technol 1996; 56(7): 749-754.

21. Weng GJ and Sun CT. Effects of fiber length on the elastic moduli of randomly oriented chopped-fiber composites. In: Tsai SW (ed.) The 5th Conference on Composite Materials, New Orleans, 20-22 March 1978, pp.149-162, ASTM-STP-674.

22. Weissenbek $\mathrm{E}$ and Rammerstorfer FG. Influence of the fiber arrangement on the mechanical and thermomechanical behavior of short fiber reinforced MMCs. Acta Metall Mater 1993; 41(10): 2833-2843.

23. Gao HJ, Ji BH, Jager IL, et al. Materials become insensitive to flaws at nanoscale: lessons from nature. Proc Natl Acad Sci USA 2003; 100(10): 5597-5600.
24. Zhang ZQ, Liu B, Huang Y, et al. Mechanical properties of unidirectional nanocomposites with non-uniformly or randomly staggered platelet distribution. J Mech Phys Solids 2010; 58(10): 1648-1660.

25. Cox HL. The elasticity and strength of paper and other fibrous materials. Br J Appl Phys 1952; 3(3): 72-79.

26. Waters JF, Lee $\mathrm{S}$ and Guduru PR. Mechanics of axisymmetric wavy surface adhesion: JKR-DMT transition solution. Int J Solids Struct 2009; 46(5): 1033-1042.

27. Schadler LS, Laird C, Melanitis N, et al. Interfacial studies on carbon/thermoplastic model composites using laser Raman spectroscopy. J Mater Sci 1992; 27(6): 1663-1671.

28. Ji BH and Gao HJ. Mechanical properties of nanostructure of biological materials. J Mech Phys Solids 2004; 52(9): 1963-1990.

29. Kelly A and Tyson WR. Tensile properties of fibre-reinforced metals: copper/tungsten and copper/molybdenum. J Mech Phys Solids 1965; 13(6): 329-338.

30. Zuo SC and Wei YG. Effective elastic modulus of bonelike hierarchical materials. Acta Mech Solida Sin 2007; 20(3): 198-205.

31. Yuan F, Stock SR, Haeffner DR, et al. A new model to simulate the elastic properties of mineralized collagen fibril. Biomech Model Mechanobiol 2011; 10(2): 147-160.

32. Zhao YH and Weng GJ. Effective elastic moduli of ribbon reinforced composites. J Appl Mech ASME 1990; 57(1): 158-167. 
